\title{
Short communication: Behavioral attitude scores associated with bovine respiratory disease identified using calf lung ultrasound and clinical respiratory scoring
}

\author{
M. C. Cramer, ${ }^{1}$ K. L. Proudfoot ${ }^{2}$ and T. L. Ollivett ${ }^{3 *}$ \\ ${ }^{1}$ Department of Dairy Science, University of Wisconsin, Madison 53706 \\ ${ }^{2}$ Department of Veterinary Preventive Medicine, The Ohio State University, Columbus 43210 \\ ${ }^{3}$ Department of Medical Sciences, School of Veterinary Medicine, University of Wisconsin, Madison 53706
}

\section{ABSTRACT}

The objective of this study was to determine whether calves exhibit differences in behavioral attitude when diagnosed with their first bovine respiratory disease (BRD) event and whether fever $\left(\geq 39^{\circ} \mathrm{C}\right)$ at the time of BRD diagnosis affected attitude. Preweaned dairy calves ( $\mathrm{n}=280 ; 21 \pm 6 \mathrm{~d}$ ) were examined twice weekly until weaning using a clinical respiratory score (CRS; CRS+: 2 respiratory categories with scores of 2 or greater; CRS-: 1 respiratory category with a score of 2 or greater or all respiratory categories scoring less than 2), lung ultrasound, and attitude score (normal = bright, alert, responsive; depressed $=$ dull but responds to stimulation, slow to stand, or reluctant to lie down). Bovine respiratory disease was categorized as subclinical BRD (SBRD; CRS- and lung consolidation $\geq 1 \mathrm{~cm}^{2}$; $\mathrm{n}=164)$ or clinical BRD (CBRD; CRS+, with or without lung consolidation; $\mathrm{n}=79$ ). Calves without $\mathrm{BRD}$ (NOBRD; $\mathrm{n}=37$ ) remained CRS- with lung consolidation $<1 \mathrm{~cm}^{2}$ for the study. Depressed attitudes were found in 23, 6, and $0 \%$ of CBRD, SBRD, and NOBRD calves, respectively. In calves with $\mathrm{CBRD}$, the odds of having a depressed attitude were 5.2 (95\% confidence interval, confidence interval: $1.1-23.7)$ and $4.5(95 \%$ confidence interval: 2.0-10.4) times higher compared with the odds of NOBRD and SBRD calves having a depressed attitude, respectively. The odds of having a depressed attitude did not differ between SBRD and NOBRD calves. Fever was associated with the odds of having a depressed attitude score, whereby calves with a fever had 6.2 (95\% confidence interval: 2.8-14) times higher odds of having a depressed attitude score compared with calves without a fever. Sensitivity and specificity of the attitude score for identifying CBRD were $23 \%$ (95\% confidence interval: 14-33) and 95\%

Received August 13, 2018.

Accepted March 10, 2019

*Corresponding author: ollivett@wisc.edu
(95\% confidence interval: 82-99), respectively. Producers should be cautious when using this attitude score as the primary means of detecting calves affected by BRD.

Key words: automated calf feeder, calf lung ultrasound, pneumonia, welfare

\section{Short Communication}

Bovine respiratory disease (BRD) is a predominant cause of preweaned dairy calf morbidity and mortality (USDA, 2010). Early detection is challenging (Buczinski et al., 2015; Cramer et al., 2016; Ollivett and Buczinski, 2016), particularly when calves are housed in groups where it is difficult to observe individual animals. Clinical scoring systems for BRD that rely solely on visual signs and rectal temperature lack sensitivity (Buczinski et al., 2014) and preclude our ability to accurately identify all affected calves. Conversely, calf lung ultrasound is a sensitive indicator of $\mathrm{BRD}$ regardless of the clinical status of the calf (Ollivett and Buczinski, 2016) and can be combined with a clinical scoring system to improve identification of BRD. However, there is a need to easily determine sensitive indicators of BRD in calves on-farm without expensive equipment.

Some visual behavioral changes have been assessed for their ability to convey clinical BRD (Cramer et al., 2016), but little is known about the behavior of calves with subclinical BRD. The Wisconsin Calf Health Scoring App (https://www.vetmed.wisc.edu/ $\mathrm{dms} /$ fapm/apps/chs.htm) includes an attitude score, a measure of calf behavior, to be used when screening calves for disease. Although performed simultaneously, this attitude score is separate from the clinical respiratory score (CRS; McGuirk and Peek, 2014) and might serve as a fast and cost-effective way to find sick calves. Therefore, the objective of this cohort study was to determine whether calves with $\mathrm{BRD}$, diagnosed by lung ultrasound and CRS, exhibit differences in behavioral attitude on the day of diagnosis. 
Calves in the present study were from the same population of calves as in Cramer and Ollivett (2019). Data collection for this cohort study took place in Ohio between February and August 2016 on a facility that raised dairy heifer and bull calves and freshened only nulliparous heifers. The Institutional Animal Care and Use Committee at the University of Wisconsin-Madison (A005049-A03) approved this study. Animal management, illness treatments, enrollment criteria, and the collection of health data have been described by Cramer and Ollivett (2019).

Attitude score, CRS, and lung ultrasound scores were assigned twice weekly between enrollment and $50 \mathrm{~d}$ of age. Attitude was scored using the criteria from the Wisconsin Calf Health Scoring App $(0=$ bright, alert, responsive; $1=$ dull but responds to stimulation; $2=$ depressed, slow to stand or reluctant to lie down; $3=$ unresponsive to stimulation; https://www.vetmed.wisc .edu/dms/fapm/apps/chs.htm). The attitude score was assigned before the CRS and lung ultrasound. More specifically, resting calves were stimulated to rise and move to the corner of the pen using the following actions in order: vocal cue, light touch on the back, or vigorously running of hands up and down the spine. Standing calves were gently guided to the corner of the pen using a vocal cue or a light touch on the hindquarters.

Calves were considered CRS positive $(\mathbf{C R S}+)$ when at least 2 respiratory categories (nose, eyes, ears, cough, rectal temperature) scored 2 or greater (McGuirk and Peek, 2014). Clinical respiratory score negative (CRS-) was defined as 1 respiratory category with a score of 2 or greater or all respiratory categories scoring less than 2. Calves were positive for lung consolidation when $\geq 1$ $\mathrm{cm}^{2}$ of lung consolidation was detected. Diarrhea was defined as fecal score $\geq 2$ (loose but stays on top of bedding or watery and sifts through bedding; McGuirk, 2008). Fever was defined as rectal temperature $\geq 39^{\circ} \mathrm{C}$ (McGuirk, 2008). Three research staff members performed the CRS, whereas only 1 researcher performed the ultrasound exams and attitude scores. Scores were recorded calf-side in the Wisconsin Calf Health Scoring App. Details regarding standardized treatment protocols for CRS+ calves, lung ultrasound technique, and the interobserver agreement for CRS status are reported in Cramer and Ollivett (2019).

Data were analyzed using SAS (version 9.3; SAS Institute Inc., Cary, NC). The experimental unit was the calf. The predictor of interest was BRD status based on the calf's first BRD event (Cramer and Ollivett, 2019): clinical BRD (CBRD), defined as CRS+ at the first BRD event and lung consolidation $<1 \mathrm{~cm}^{2}$; subclinical BRD (SBRD), defined as CRS - and lung consolidation $\geq 1 \mathrm{~cm}^{2}$ at the first BRD event; or without BRD (NO-
BRD), defined as never being CRS+ and never having lung consolidation $\geq 1 \mathrm{~cm}^{2}$. The outcome of interest was the attitude score on the day of BRD diagnosis (29 \pm $8 \mathrm{~d}$ ) or the attitude score on the age-equivalent day for NOBRD calves (assigned on or immediately adjacent to d 29). A 1-way ANOVA was used to ensure that age at the attitude score was similar between BRD statuses. Fecal scores and rectal temperatures on the day of BRD diagnosis for calves with CBRD and SBRD or at the time of attitude score for NOBRD calves were used to define diarrhea and fever, respectively. The distribution of BRD status within our original attitude score 1 and attitude score 2 were similar (Fisher's exact test; $P=$ 0.1761 ); therefore, we collapsed these 2 categories into 1 category called "depressed." For analysis, attitude score was defined as a dichotomous variable (normal attitude and depressed attitude). Calves entering the automated calf feeder barn at $\geq 30 \mathrm{~d}$ of age and NOBRD calves that left the barn before $50 \mathrm{~d}$ were excluded from final analyses.

Criteria for variable inclusion in the multivariable model are described in Cramer and Ollivett (2019) and followed Dohoo et al. (2003) for detecting potential confounders. One logistic regression model (PROC GLIMMIX) was used to determine whether BRD status was associated with attitude score on the day of BRD diagnosis. A separate logistic model was used to determine whether fever at the time of BRD diagnosis was associated with attitude score on the day of BRD diagnosis. Initial multivariable models included BRD status or fever and dystocia (no assistance or easy pull $=0$; hard pull or surgical delivery $=1$ ), season of birth (calves born on or before February 29, $2016=$ winter; calves born between March 1 and April 30, $2016=$ spring; calves born on or after May 1, $2016=$ summer), and diarrhea as fixed effects. The final model included only BRD status. Predicted probabilities were assessed using the LSMEANS statement, and differences between BRD statuses were assessed using differences in LSMEANS. Measures of central tendency for raw data are presented as mean \pm standard deviation or median (first quartile, third quartile) after assessing normality. Estimates from multivariable models are presented as mean \pm standard error. Significance was defined as $\alpha<0.05$. Where attitude score and BRD status were found to be associated, sensitivity and specificity of the attitude score for detecting BRD was calculated as described by Dohoo et al. (2003). Attitude score was treated as the new test, and BRD status was the gold standard.

A total of 280 calves were enrolled and included in the analyses. These calves were $21 \pm 6 \mathrm{~d}$ of age (mean $\pm \mathrm{SD}$ ) at enrollment and were housed in groups of 13 \pm 3 . A description of the number of calves born at this 
facility during the study period and excluded from the study is included in Cramer and Ollivett (2019). Calf descriptive information and the proportion of calves with diarrhea and fever at the time of BRD diagnosis are presented in Table 1 . Twenty-eight percent $(79 / 280)$ and $59 \%(164 / 280)$ of calves were identified as having CBRD and SBRD at their first BRD event, respectively. Thirteen percent $(37 / 280)$ of calves were categorized as NOBRD. The age of calves with NOBRD $(33 \pm 5$ d), CBRD $(27 \pm 9 \mathrm{~d})$, and SBRD $(30 \pm 8 \mathrm{~d})$ on the day of diagnosis was not different between BRD groups $(P$ $=0.73$ ). The raw proportion of calves with normal and depressed attitude scores on the day of their first BRD diagnosis is presented in Table 2 .

Table 1. Proportion [\% (no.)] of calves in each category of dystocia and season of birth by bovine respiratory disease (BRD) status and proportion of calves with diarrhea and fever at the time of BRD diagnosis by BRD status ${ }^{1}$

\begin{tabular}{|c|c|c|c|}
\hline \multirow[b]{2}{*}{ Variable (no.) } & \multicolumn{3}{|c|}{ BRD status $^{2}$} \\
\hline & $\begin{array}{c}\text { CBRD } \\
(\mathrm{n}=79)\end{array}$ & $\begin{array}{c}\text { SBRD } \\
(\mathrm{n}=164)\end{array}$ & $\begin{array}{l}\text { NOBRD } \\
(\mathrm{n}=37)\end{array}$ \\
\hline \multicolumn{4}{|l|}{ Dystocia $^{3}$} \\
\hline With & $16(13)$ & $20(32)$ & $5(2)$ \\
\hline Without & $84(66)$ & $80(132)$ & $95(35)$ \\
\hline \multicolumn{4}{|c|}{ Season of birth ${ }^{4}$} \\
\hline Winter & $59(47)$ & $37(61)$ & $16(6)$ \\
\hline Spring & $22(17)$ & $26(42)$ & $30(11)$ \\
\hline Summer & $19(15)$ & $37(61)$ & $54(20)$ \\
\hline \multicolumn{4}{|l|}{ Diarrhea $^{5}$} \\
\hline With & $28(22)$ & $13(21)$ & $14(5)$ \\
\hline Without & $72(57)$ & $87(143)$ & $86(32)$ \\
\hline \multicolumn{4}{|l|}{ Fever $^{6}$} \\
\hline With & $51(40)$ & $27(44)$ & $0(0)$ \\
\hline Without & $49(39)$ & $73(120)$ & $100(37)$ \\
\hline
\end{tabular}

${ }^{1}$ Calves were enrolled in the study at (mean \pm SD) $21 \pm 6 \mathrm{~d}$ of age and underwent twice-weekly health exams.

${ }^{2}$ Researchers performed twice-weekly health exams, which included a clinical respiratory score (CRS; CRS+: 2 respiratory categories with scores of 2 or greater; CRS-: 1 respiratory category with a score of 2 or greater or all respiratory categories scoring less than 2; calculated with nasal, eye, ear, cough, and rectal temperature scores; McGuirk and Peek, 2014) and a lung ultrasound score (0 to 5, based on severity of lung consolidation; Ollivett and Buczinski, 2016). The BRD status for each calf was defined as subclinical BRD (SBRD; calves with any lung consolidation $\geq 1 \mathrm{~cm}^{2}$ and CRS-) or clinical BRD (CBRD; CRS+ with or without lung consolidation) based on the first BRD event. Calves without BRD (NOBRD) never had lung consolidation $\geq 1 \mathrm{~cm}^{2}$ or CRS+.

${ }^{3} \mathrm{With}=$ hard pull or surgical delivery; without $=$ no assistance or easy pull.

${ }^{4}$ Winter $=$ calves born on or before February 29, 2016; spring $=$ calves born between March 1 and April 30, 2016; summer = calves born on or after May 1, 2016.

${ }^{5}$ Diarrhea at the time of BRD diagnosis, defined as fecal score $\geq 2$ : $(0$ $=$ normal fecal consistency; $1=$ semiformed, pasty; $2=$ loose but stays on top of bedding; 3 = watery, sifts through bedding; McGuirk, 2008). For NOBRD calves, fecal scores from $29 \mathrm{~d}$ of age were used.

${ }^{6} \mathrm{Rectal}$ temperature $\geq 39^{\circ} \mathrm{C}$ at the time of BRD diagnosis. For NOBRD calves, rectal temperatures from $29 \mathrm{~d}$ of age were used.
Table 2. Proportion [\% (no.)] of calves with normal or depressed attitude scores at their first bovine respiratory disease (BRD) event by BRD status ${ }^{1}$

\begin{tabular}{lccc}
\hline & \multicolumn{3}{c}{ BRD status $^{3}$} \\
\cline { 2 - 4 } $\begin{array}{l}\text { Attitude } \\
\text { score }^{2}\end{array}$ & $\begin{array}{c}\text { CBRD } \\
(\mathrm{n}=79)\end{array}$ & $\begin{array}{c}\text { SBRD } \\
(\mathrm{n}=164)\end{array}$ & $\begin{array}{c}\text { NOBRD } \\
(\mathrm{n}=37)\end{array}$ \\
\hline $\begin{array}{l}\text { Normal } \\
\text { Depressed }\end{array}$ & $\begin{array}{c}77(61) \\
23(18)\end{array}$ & $\begin{array}{c}94(154) \\
6(10)\end{array}$ & $\begin{array}{c}5(35) \\
5(2)\end{array}$
\end{tabular}

${ }^{1}$ Calves were enrolled in the study at (mean \pm SD) $21 \pm 6 \mathrm{~d}$ of age and underwent twice-weekly health exams.

${ }^{2}$ Attitude score obtained from the Wisconsin Calf Health Scoring App (https://www.vetmed.wisc.edu/dms/fapm/apps/chs.htm): normal = bright, alert, responsive; depressed $=$ dull but responds to stimulation, slow to stand, or reluctant to lie down. We determined the mean age of all calves with SBRD and CBRD ( $29 \pm 8 \mathrm{~d}$ of age); for the NOBRD calves, we selected the attitude score from a health examination day that most closely corresponded with this mean age.

${ }^{3}$ Researchers performed twice-weekly health exams, which included a clinical respiratory score (CRS; CRS+: 2 respiratory categories with scores of 2 or greater; CRS-: 1 respiratory category with a score of 2 or greater or all respiratory categories scoring less than 2; McGuirk and Peek, 2014) and lung ultrasound to determine lung consolidation. The BRD status for each calf was defined as subclinical BRD (SBRD; calves with any lung consolidation $\geq 1 \mathrm{~cm}^{2}$ and $\mathrm{CRS}-$ ) or clinical BRD (CBRD; CRS+ with lung consolidation $<1 \mathrm{~cm}^{2}$ ) based on the first BRD event. Calves without BRD (NOBRD) never had lung consolidation $\geq 1 \mathrm{~cm}^{2}$ or CRS+.

The multivariable logistic model showed an effect of BRD status on the odds of having a depressed attitude score on the day of BRD diagnosis $(P<0.001)$. The mean predicted probability of having a depressed attitude, based on BRD status, is shown in Figure 1. In calves with CBRD, the odds of having a depressed attitude score were 5.2 (95\% CI: 1.1-23.7) and 4.5 (95\% CI: 2.0-10.4) times higher compared with the odds of having a depressed attitude score in calves with NO$\operatorname{BRD}(P=0.0350)$ and calves with SBRD $(P<0.001)$, respectively. There was no difference in the odds of having a depressed attitude score between calves with SBRD and NOBRD $(P=0.87)$. Fever was associated with the odds of having a depressed attitude score, whereby calves with a fever had 6.2 (95\% CI: 2.8-14) times higher odds of having a depressed attitude score compared with calves without a fever $(P<0.0001)$. The sensitivity and specificity of the attitude score to identify calves with CBRD were 23\% (95\% CI: 14-33) and $95 \%$ (95\% CI: 82-99), respectively.

To our knowledge, this study was the first to investigate the associations between behavioral attitude score and BRD using calf lung ultrasound and clinical respiratory scoring for BRD diagnosis. Calves with CBRD were more likely to have a depressed attitude score compared with calves with SBRD and calves with NOBRD. However, the attitude score had a poor sensitivity and would identify only $23 \%$ of clinical cases if used alone. 
Fever was most common in CBRD calves and may be driving the relationship between attitude score and BRD status. Calves with fever had higher odds of having a depressed attitude when analyzed separately from BRD, providing more evidence that suggests fever strongly influences attitude score. Fever develops in response to inflammatory cytokines released during infection (Kluger, 1991). These cytokines, such IL-1, IL-6, and $\mathrm{TNF}-\alpha$, are also responsible for altering muscle metabolism and may directly and indirectly reduce a calf's desire to interact in the environment, which may be interpreted as depression (Hart, 1988). These findings suggest that clinical calves experience a more systemic inflammatory process, at least at the time of diagnosis, than subclinical calves. It may fall to reason, then, that despite obvious lung level differences in inflammation, the systemic inflammatory process may be similar between subclinical and unaffected calves (Ollivett et al.,

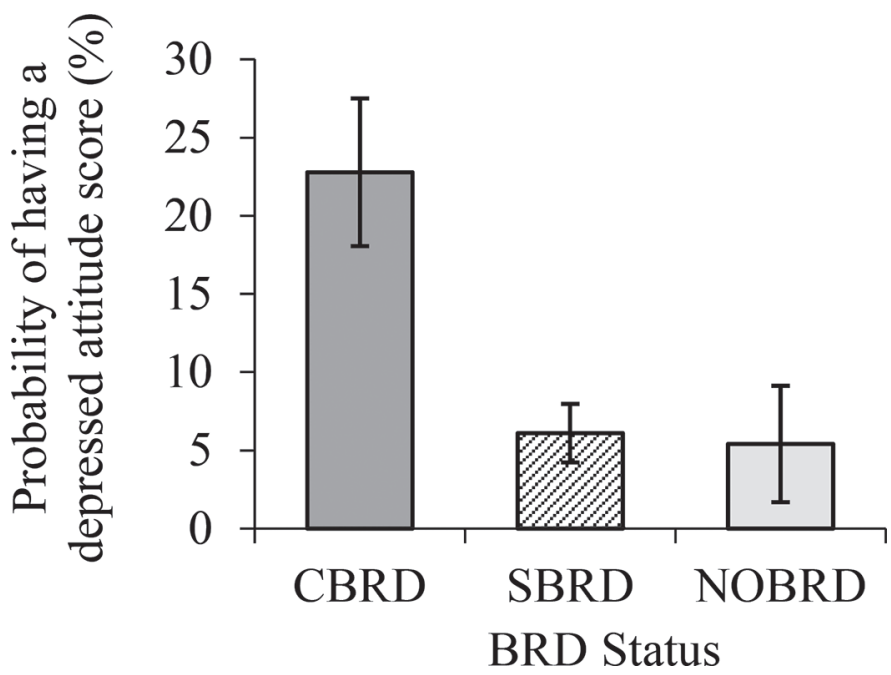

Figure 1. The predicted probabilities (LSM \pm SEM) of having a depressed attitude score by bovine respiratory disease (BRD) status $(P$ $=0.0008$ ) using PROC GLIMMIX in SAS (version 9.3; SAS Institute Inc., Cary, NC). Attitude scores were obtained from the Wisconsin Calf Health Scoring App (https://www.vetmed.wisc.edu/dms/fapm/ apps (chs.htm). Normal $=$ bright, alert, and responsive; depressed $=$ dull but responds to stimulation, slow to stand, or reluctant to lie down. We determined the mean age of all calves with subclinical BRD (SBRD) and clinical BRD (CBRD; $29 \pm 8 \mathrm{~d}$ of age) and the calves without BRD (NOBRD) and selected the attitude score from a health examination day that most closely corresponded with this mean age. The BRD status was determined from twice-weekly health exams, which included a clinical respiratory score [CRS; CRS+: 2 respiratory categories (nose, eyes, ears, cough, and rectal temperature) with scores of 2 or greater; CRS-: 1 respiratory category with a score of 2 or greater or all respiratory categories scoring less than 2; McGuirk and Peek, 2014] and a lung ultrasound to determine lung consolidation. The BRD status for each calf was defined as CBRD ( $\mathrm{n}=79$; CRS + with lung consolidation $\left.<1 \mathrm{~cm}^{2}\right)$ or SBRD $(\mathrm{n}=64$; CRS- with lung consolidation $\geq 1 \mathrm{~cm}^{2}$ ) based on the first BRD event. Calves with NOBRD $(\mathrm{n}=37)$ never had lung consolidation $\geq 1 \mathrm{~cm}^{2}$ or CRS+.
2015). Previous studies found less exploratory behavior (Cramer and Stanton, 2015), rumination, hay eating, and self-grooming (Borderas et al., 2008) in response to clinical disease or an LPS challenge compared with unaffected calves. However, future studies that directly compare the concentration of inflammatory cytokines, behavior, and BRD status as diagnosed by lung ultrasound and clinical respiratory scoring could help us understand why some calves with BRD show clinical signs and others do not.

The sensitivity of this attitude score to detect calves with CBRD was much lower than other currently available tools, such as a previously proposed behaviorbased screening tool (Cramer et al., 2016), the CRS (McGuirk and Peek, 2014), and calf lung ultrasound (Buczinski et al., 2014). The sensitivity of the attitude score might have been low due to our study design and the use of thoracic ultrasound and CRS. By examining calves twice weekly with relatively sensitive tools, we detected new cases of disease very early after onset, possibly before enough time had elapsed for calves to develop a systemic inflammatory response that was severe enough to display outward signs of depressed attitude. It is possible that the sensitivity of attitude score could be higher in calves that are evaluated less frequently or with less sensitive tools because the underlying disease and systemic manifestations are more progressed at diagnosis. It is also possible that including other behaviors in the attitude score, such as isolation from the group (e.g., Cramer et al., 2016), may have improved our ability to correctly classify calves based on behavior.

Lung consolidation (Binversie, 2017) and CBRD (Cramer and Stanton, 2015) have been reported in calves less than 3 wk of age. Our study was limited in that calves were enrolled at approximately $21 \mathrm{~d}$ of age, and those identified as normal may have had BRD in the past but recovered before study enrollment. Furthermore, the researcher that assigned the attitude score also assigned clinical scores for some calves and lung ultrasound scores for all calves. Having an awareness of the attitude score could have influenced the clinical or ultrasound score assigned by the researcher, potentially increasing the number of calves classified with BRD. It is also possible that clinical signs (e.g., eye or nasal discharge) were noticeable before assigning the attitude score, which could have contributed to bias in this study.

Our results suggest that producers managing well-fed calves in group housing should be cautious of using this attitude score as their primary means of detecting BRD in 3- to 6-wk-old calves. Calves with SBRD and many calves with CBRD will go undetected unless an additional screening tool is used. 


\section{ACKNOWLEDGMENTS}

This work was funded by STGenetics (Navasota, TX). Any opinions, findings, conclusions, or recommendations expressed in this publication are those of the authors and do not necessarily reflect the view of STGenetics. The authors thank the staff and owners of the facility and Ella Jackson and Hannah Donley (The Ohio State University, Columbus) for their assistance.

\section{REFERENCES}

Binversie, E. 2017. Using visual observations and thoracic ultrasonography to evaluate treatment success in preweaned dairy calves affected by respiratory disease. MSc Thesis. University of Wisconsin, Madison.

Borderas, T. F., A. M. de Passillé, and J. Rushen. 2008. Behavior of dairy calves after a low dose of bacterial endotoxin. J. Anim. Sci. 86:2920-2927.

Buczinski, S., G. Forté, D. Francoz, and A. M. Bélanger. 2014. Comparison of thoracic auscultation, clinical score, and ultrasonography as indicators of bovine respiratory disease in preweaned dairy calves. J. Vet. Intern. Med. 28:234-242.

Buczinski, S., T. L. Ollivett, and N. Dendukuri. 2015. Bayesian estimation of the accuracy of the calf respiratory scoring chart and ultrasonography for the diagnosis of bovine respiratory disease in pre-weaned dairy calves. Prev. Vet. Med. 119:227-231.
Cramer, M. C., and T. L. Ollivett. 2019. Growth of preweaned, grouphoused dairy calves diagnosed with respiratory disease using clinical respiratory scoring and thoracic ultrasound-A cohort study. J. Dairy Sci. 102:4322-4331.

Cramer, M. C., T. Ollivett, and A. Stanton. 2016. Associations of behavior-based measurements and clinical disease in preweaned, group-housed dairy calves. J. Dairy Sci. 99:7434-7443.

Cramer, M. C., and A. Stanton. 2015. Associations between health status and the probability of approaching a novel object or stationary human in preweaned group-housed dairy calves. J. Dairy Sci. 98:7298-7308.

Dohoo, I., W. Martin, and H. Stryhn. 2003. Veterinary Epidemiologic Research. AVC Inc., Charlottetown, PEI, Canada.

Hart, B. L. 1988. Biological basis of the behavior of sick animals. Neurosci. Biobehav. Rev. 12:123-137.

Kluger, M. J. 1991. Fever: Role of pyrogens and cryogens. Physiol. Rev. 71:93-127.

McGuirk, S. M. 2008. Disease management of dairy calves and heifers. Vet. Clin. North Am. Food Anim. Pract. 24:139-153.

McGuirk, S. M., and S. F. Peek. 2014. Timely diagnosis of dairy calf respiratory disease using a standardized scoring system. Anim. Health Res. Rev. 15:145-147.

Ollivett, T., and S. Buczinski. 2016. On-farm use of ultrasonography for bovine respiratory disease. Vet. Clin. North Am. Food Anim. Pract. 32:19-35.

Ollivett, T., J. Caswell, D. Nydam, T. Duffield, K. Leslie, J. Hewson, and D. Kelton. 2015. Thoracic ultrasonography and bronchoalveolar lavage fluid analysis in Holstein calves with subclinical lung lesions. J. Vet. Intern. Med. 29:1728-1734.

USDA. 2010. Heifer Calf Health and Management Practices on U.S. Dairy Operations, 2007. USDA, Fort Collins, CO. 